\title{
Up-regulation of inducible heat shock protein-70 expression in multiple sclerosis patients
}

Mansilla, M ; Comabella, M ; Río, J ; Castilló, J ; Castillo, M ; Martin, R ; Montalban, X ; Espejo, C

\begin{abstract}
Inducible heat shock protein (HSP)70 (HSP70-1A and HSP70-1B proteins) is a chaperone responsible for assisting proper protein folding. Following stress conditions, HSP70 is highly up-regulated to mediate cytoprotective functions. In addition, HSP70 is able to trigger innate and adaptive immune responses that promote the immune recognition of antigens and to act as a cytokine when it is released. The data in the literature are controversial with regard to expression studies in peripheral blood mononuclear cells (PBMCs). In the present study, we aimed to examine if alterations of HSP70-1A/B expression are involved in the autoimmune pathogenesis of multiple sclerosis (MS). We determined both mRNA and protein expression in PBMCs of MS patients and healthy donors (HDs). We found a baseline increased expression of the HSPA1A gene in PBMCs from MS patients compared with HDs. Gene expression findings were associated with an increased protein expression of HSP70-1A/B in T lymphocytes (CD4+ and CD8+) and monocytes from MS patients under basal conditions that may reflect the immunological activation occurring in MS patients. We also provided evidence that heat shock (HS) stimulus induced HSP70-1A/B protein expression in HDs and MS patients, and that HS-induced HSP70-1A/B protein expression in monocytes correlated with the number of T2 lesions at baseline in MS patients. However, after lipopolysaccharide inflammatory stimulus, monocytes from MS patients failed to induce HSP70-1A/B protein expression. Our data hint at altered immune responses in MS and may indicate either a state of chronic stress or increased vulnerability to physiological immune responses in MS patients.
\end{abstract}

DOI: https://doi.org/10.3109/08916934.2013.866104

Posted at the Zurich Open Repository and Archive, University of Zurich

ZORA URL: https://doi.org/10.5167/uzh-89024

Journal Article

Accepted Version

Originally published at:

Mansilla, M; Comabella, M; Río, J; Castilló, J; Castillo, M; Martin, R; Montalban, X; Espejo, C (2014). Up-regulation of inducible heat shock protein-70 expression in multiple sclerosis patients. Autoimmunity, 47(2):127-133.

DOI: https://doi.org/10.3109/08916934.2013.866104 


\section{Up-regulation of Inducible Heat Shock Protein-70 Expression in Multiple \\ Sclerosis Patients}

María José Mansilla ${ }^{1}$,

Manuel Comabella ${ }^{1}$,

Jordi Río ${ }^{1}$,

Joaquín Castilló ${ }^{1}$,

Mireia Castillo",

Roland Martin ${ }^{2}$,

Xavier Montalban ${ }^{1}$,

Carmen Espejo ${ }^{1}$,

${ }^{1}$ Servei de Neurologia-Neuroimmunologia. Centre d'Esclerosi Múltiple de Catalunya (CEM-Cat). Vall d'HebronInstitut de Recerca (VHIR). Hospital Universitari Vall d'Hebron. Universitat Autònoma de Barcelona, Barcelona, Spain.

${ }^{2}$ Department of Neuroimmunology and Multiple Sclerosis Research, Neurology Clinic, University Hospital Zürich, Switzerland.

Corresponding author: Carmen Espejo. Unitat de Neuroimmunologia Clínica. Centre d’Esclerosi Múltiple de Catalunya (CEM-Cat). Vall d'Hebron Institut de Recerca. Ps. Vall d'Hebron, 119-129, 08035 Barcelona, Spain. Phone: +34934893599. Fax: +34932746084. e-mail: carmen.espejo@vhir.org

Running head: Hsp70 in MS patients

Keywords: Heat shock protein, HSPA1A, multiple sclerosis, immune regulation, autoimmunity 


\section{Abstract}

Inducible heat shock protein (HSP)70 (HSP70-1A and HSP70-1B proteins) is a chaperone responsible for assisting proper protein folding. Following stress conditions, HSP70 is highly up-regulated to mediate cytoprotective functions. In addition, HSP70 is able to trigger innate and adaptive immune responses that promote the immune recognition of antigens and to act as a cytokine when it is released.

The data in the literature are controversial with regard to expression studies in peripheral blood mononuclear cells (PBMCs). In the present study, we aimed to examine if alterations of $\mathrm{HSP} 70-1 \mathrm{~A} / \mathrm{B}$ expression are involved in the autoimmune pathogenesis of multiple sclerosis (MS). We determined both mRNA and protein expression in PBMCs of MS patients and healthy donors (HDs). We found a baseline increased expression of the HSPA1A gene in PBMCs from MS patients compared with HDs. Gene expression findings were associated with an increased protein expression of HSP70-1A/B in T lymphocytes (CD4+ and CD8+) and monocytes from MS patients under basal conditions that may reflect the immunological activation occurring in MS patients. We also provided evidence that heat shock (HS) stimulus induced HSP701A/B protein expression in HDs and MS patients, and that HS-induced HSP70-1A/B protein expression in monocytes correlated with the number of T2 lesions at baseline in MS patients. However, after lipopolysaccharide inflammatory stimulus monocytes from MS patients failed to induce HSP70-1A/B protein expression. Our data hint at altered immune responses in MS and may indicate either a state of chronic stress or increased vulnerability to physiological immune responses in MS patients. 


\section{Introduction}

Heat shock proteins (HSPs) are evolutionarily conserved chaperones that assist in the proper folding of newly synthesised proteins and are responsible for the quality control of misfolded proteins. Consequently, HSPs play an essential role in promoting cell survival following stress or harmful conditions such as heat shock, nutrient deprivation, irradiation, hypoxia, oxidative and toxic stresses, infection, and exposure to inflammatory cytokines. Under such stress conditions, inducible HSPs are highly upregulated as the result of a heat shock response to maintain cellular homeostasis and to facilitate cell survival functions (1-5).

Among the HSPs, HSP70 is the most conserved and intensely studied family and includes the constitutively expressed HSC70 (HSP73) and the stress-inducible HSP70 (HSP70-1A and HSP70-1B) proteins (6-8). HSP70 is located mainly in the cytosol. Adverse conditions trigger HSP70 expression, which then translocates to the nucleus and activates cytoprotective functions, acting as a chaperone and anti-apoptotic mediator. In addition to intracellular cytoprotective roles, HSP70 can be released actively via exosomes to the extracellular milieu, where it acts as a danger signal that induces immune responses. Furthermore, HSP70 can participate in the uptake and cross-presentation of antigens, and this role has been employed to improve tumorspecific vaccination protocols $(9-10)$. The above brief summary is far from complete, but indicates that HSP70 could be involved in chronic inflammatory and autoimmune conditions at multiple levels and by several different mechanisms.

MS is considered a prototypic $T$ cell-mediated autoimmune disease that is characterised by inflammation, and various degrees of demyelination, damage of axons and neurons and glial scarring in the central nervous system (CNS). The currently approved therapies of MS are all immunomodulatory or immunosuppressive, and neuroprotective approaches are being examined, but not yet available to patients. In CNS lesions in both MS patients and animals with experimental autoimmune encephalomyelitis (EAE), the prototypic animal model for $M S$, an up-regulation of 
HSP70 expression has been observed and interpreted as activation of endogenous neuroprotective mechanisms (11-13). In contrast, Cwiklinska and co-workers (2003) detected HSP70-myelin basic protein (MBP) and HSP70-proteolipid protein (PLP) complexes in the CNS lesions of MS patients. Paradoxically, these MBP/PLP-HSP70 complexes are highly immunogenic and are able to induce specific T-cell responses in animals with EAE (13-15).

The genes coding for HSP70 (HSPA1A and HSPA1B) are located on chromosome 6 (6p21.3) within the HLA region. Gene expression studies using microarrays have reported a down-regulation of $H S P A 1 A$ expression in peripheral blood mononuclear cells (PBMCs) of MS patients compared to healthy donors (HDs) (16-19). Although histopathological studies have demonstrated an up-regulation of HSP70 in MS and EAE lesions, protein analyses of HSP70 expression in PBMCs did not reveal differences between MS patients and HDs (20). Despite the absence of differences in HSP70 protein expression in freshly isolated PBMCs, an increased induction of HSP70 expression was observed in PBMCs of MS patients following an inflammatory or heat shock stimulus (20). The above data from previous studies as well as the known functions of HSP70 indicate that HSP70 itself and/or other family members of this fundamental cytoprotective pathway may be involved in the autoimmune pathogenesis of MS.

Based on the above in part controversial observations, we aimed to investigate the potential role of HSP70 in MS pathogenesis by determining its gene and protein expression under different experimental conditions in a well-characterized cohort of relapse-onset MS patients and age- and gender-matched HDs. 


\section{Methods}

Patients

Patients with clinically isolated syndromes (CIS) and untreated relapsing-remitting MS (RRMS) patients were included in this study. No patient had clinical exacerbations or had received corticosteroid treatment during the month prior to blood sample collection. An age- and gender-matched control group of HDs was also included. All patients and HDs gave their informed consent, and this study was approved by the local ethics committee. Demographic and baseline clinical characteristics of the MS patients and HDs included in the study are shown in Table 1.

\section{Sample collection}

Blood samples from MS patients and HDs were collected by standard venipuncture in sodium citrate tubes, and PBMCs were isolated by Ficoll-Isopaque density gradient centrifugation (Gibco BRL, Life Technologies LTD, UK) according to standard procedures. Isolated PBMCs were re-suspended in RPMI 1640 culture medium supplemented with 10\% heat-inactivated foetal bovine serum (FBS), $2 \mathrm{mM}$ glutamine, $25 \mathrm{mM}$ HEPES, $50 \mathrm{U} / \mathrm{ml}$ of penicillin, and $50 \mathrm{mg} / \mathrm{ml}$ of streptomycin, all obtained from Gibco BRL (Paisley, UK). Aliquots of PBMCs from a subgroup of MS patients and HDs were cryopreserved and stored in liquid nitrogen.

\section{HSP70 gene expression}

HSPA1A gene expression was analysed in fresh PBMCs (30 MS patients and 30 HDs) and in a subgroup of cryopreserved PBMC samples (9 MS patients and $10 \mathrm{HDs}$ ). Total RNA was obtained from both fresh and frozen PBMCs using an RNeasy Mini Kit (Qiagen, Germany). One microgram of RNA was retrotranscribed into cDNA using an Omniscript RT kit (Qiagen), and the gene expression levels of HSPA1A were assessed by real-time PCR relative quantification using Taqman expression assays (Applied Biosystems, CA, USA). PGK1 (phosphoglycerate-kinase 1; Applied Biosystems) was 
used as an endogenous control. To select the appropriate endogenous control for data normalisation, 11 endogenous genes were previously tested using the Taqman human endogenous control plate (Applied Biosystems) in four PBMC samples from both MS patients and HDs. Real-time PCR was performed in an ABI Prism 7000 thermal cycler (Applied Biosystems) according to manufacturer's instructions. The threshold cycle $\left(\mathrm{C}_{\mathrm{T}}\right)$ value for each reaction was used to calculate the relative level of gene expression for each sample using the $2^{-\Delta \Delta C T}$ method (21). Samples were tested in triplicate.

\section{HSP70 protein expression}

The expression of intracellular HSP70-1A/B protein was assessed in CD4+ (CD3+CD8) and CD8+ (CD3+CD8+) T lymphocytes and monocytes (CD14+) by flow cytometry from non-stimulated PBMCs or from PBMCs stimulated by either heat shock (HS) or an inflammatory challenge (20). In brief, for the HS stimulus PBMCs were incubated at $40^{\circ} \mathrm{C}$ overnight in a water bath followed by a 30 -min recovery period at $37^{\circ} \mathrm{C}$. The inflammatory stress was induced incubating PBMCs in the presence of $5 \mu \mathrm{g} / \mathrm{ml}$ lipopolysaccharide (LPS) (Sigma Aldrich, Saint Louis, MI) for $24 \mathrm{~h}$ in humidified atmosphere at $5 \% \mathrm{CO}_{2}$ and $37^{\circ} \mathrm{C}$. Following stress stimuli, PBMCs were washed with phosphate-buffered saline (PBS) and incubated with a saturating amount of PerCP-conjugated anti-human CD3 (Becton Dickinson (BD) Pharmingen, San Diego, CA), FITC-conjugated anti-human CD8 (BD Pharmingen), and APC-conjugated antihuman CD14 (BD Pharmingen) or the corresponding isotype controls (BD Pharmingen) for $25 \mathrm{~min}$ at room temperature. Then, the cells were washed with PBS and fixed and permeabilised using two different commercial intracellular staining protocols following the manufacturer's instructions to quantify the cytoplasmic (Dako IntraStain, Fixation and Permeabilization kit; Dako, Denmark) and total (cytoplasmic plus nuclear) (BD Cytofix/Cytoperm; BD Bioscience) HSP70-1A/B protein expression. During the permeabilisation process, the cells were incubated with a PE-conjugated monoclonal 
antibody against HSP70-1A/-1B (clone C92F3A-5, reference SPA-810PE, Stressgen, Assay Designs Inc., MI), or an isotype control antibody (IgG1-PE, BD Pharmingen).

Data acquisition was performed on a FACSCanto ${ }^{\mathrm{TM}}$ flow cytometer and analysed with DIVA software (BD, Mountain View, CA). The mean fluorescence intensity (MFI) and percentage of $\mathrm{HSP} 70-1 \mathrm{~A} / \mathrm{B}$ positive cells were calculated by subtracting the isotype control signal from the HSP70-1A/B specific signal.

\section{Statistical analysis}

Data are shown as mean values (standard deviation) unless otherwise stated. Statistical analysis was performed with SPSS 17.0 software (SPSS Inc., Chicago, IL) and Prism software (GraphPad version 5.1 software Inc., San Diego, CA) both for MSWindows. Parametric and nonparametric tests were used depending on the normality conditions. To compare data from two groups, Wilcoxon, Mann-Whitney-U, or t-tests were applied. When more than two groups were compared, a Friedman test along with Dunn's post-hoc test was applied. Spearman correlations or generalized linear models were used to determine association between HSP70 expression and clinical or demographic variables of MS patients and HDs. Differences were considered statistically significant when $p<0.05$. 


\section{Results}

HSP70 gene expression is up-regulated in the PBMCs of MS patients

We first investigated differences in HSPA1A gene expression by means of real-time PCR in fresh PBMCs from 30 MS patients and $30 \mathrm{HDs}$. As shown in Figure 1A, HSPA1A gene expression was increased 2.5-fold in PBMCs of MS patients compared to HDs $(p=0.038)$. No associations of HSPA1A expression with either demographic or clinical variables of MS patients or HDs were found.

To evaluate whether sample cryopreservation could modify HSP70 gene expression, HSPA1A mRNA expression levels were also determined in a subgroup of previously frozen/thawed PBMCs from 9 MS patients and $10 \mathrm{HDs}$. As depicted in Figure 1B, HSPA1A expression did not significantly differ between fresh and cryopreserved PBMCs from MS patients or HDs.

Total HSP70-1A/B expression is increased in $T$ lymphocytes and monocytes from MS patients

We next examined HSP70-1A/B protein expression in CD4+ (CD3+CD8-) and CD8+ $(C D 3+C D 8+) T$ lymphocytes and in monocytes (CD14+) from non-stimulated PBMCs of 32 MS patients and 30 age- and gender-matched HDs. Flow cytometry analysis showed no differences in the cytoplasmic MFI expression between MS patients and HDs (Fig. 2A). Similarly, the percentage of HSP70-1A/B positive cells was comparable between groups (data not shown).

Because HSP70 translocates to the nucleus following stress conditions (22), we also analysed the total (cytoplasmic plus nuclear) cellular expression of HSP70-1A/B in a subgroup of 14 MS patients and 14 HDs. Interestingly, the PBMCs from MS patients exhibited a significant increase in total HSP70-1A/B protein expression compared to HDs in all cell types studied (for CD4, $p=0.030$; for CD8, $p=0.007$; for CD14, $p=0.003$ ) (Fig. 2B). 
Neither HSP70-1A/B T cell nor monocyte expression was associated with baseline clinical or demographic variables of MS patients or HDs.

Induction of HSP70-1A/B protein expression following HS or inflammatory stress To study the induction of HSP70-1A/B protein expression after stress stimulus, PBMCs from $14 \mathrm{MS}$ patients and $14 \mathrm{HDs}$ were challenged with a thermal $(\mathrm{HS})$ or an inflammatory (LPS) stimulus, and both the cytoplasmic and total HSP70-1A/B expression levels were analysed in T lymphocytes and in monocytes. As shown in Fig. 3, HS stress induced a total HSP70-1A/B expression in both $\mathrm{T}$ lymphocytes and monocytes in HDs (for CD4, $p<0.001$; for CD8, $p=0.013$; for CD14, $p=0.003$ ). HS also induced HSP70-1A/B expression in PBMCs from MS patients, and differences reached statistical significance for CD8+T cells $(p=0.021)$ and monocytes $(p=0.001)$ from MS patients but not in CD4+ $\mathrm{T}$ cells (Fig. 3A). In addition, we found a positive correlation between total expression of HS-induced HSP70-1A/B protein in monocytes and the number of T2 lesions at baseline in MS patients $(r=0.61 ; p=0.034)$. Interestingly, monocyte activation by LPS stimulation caused an increase in total HSP70-1A/B protein expression in HDs $(p<0.001)$ but not in MS patients (Fig. 3B). No differences were observed when cytoplasmic HSP70-1A/B expression was analysed after either HS or LPS stimulus (data not shown). 


\section{Discussion and conclusions}

Traditionally, HSP70 has been considered to be involved in homeostatic functions and cytoprotection because it participates in proper protein folding, avoidance of folding mistakes, and hence in preventing protein aggregation (23-25). In addition, it has also been reported that HSP70 blocks multiple steps of the cellular apoptosis pathway (2629). Its close location to the MHC class II region, which remains the genomic region most consistently associated with an increased genetic risk for MS (30), suggested that HSP70 may be implicated in the altered immune response that takes place in the disease. Histopathological studies in MS patients have shown an up-regulation in HSP70 expression within CNS lesions $(11-12,31)$, findings that were interpreted as a protective response of local cells to the inflammatory environment (32). Studies in peripheral blood cells, however, have revealed a lower expression of $H S P A 1 A$, one of the inducible genes for HSP70, in MS patients compared with HDs (14-16).

During recent years it has become clear that HSP70 serves a multitude of other functions than cytoprotection and could also be involved in autoantigen presentation of MBP/PLP-HSP70 complexes in MS patients (14) as well as more generally in eliciting innate and adaptive immune responses, which has recently been reviewed (33).

Based on the above previous studies on HSPA1A gene expression, the data from protein expression in CNS tissue in MS and EAE, and the broader understanding of the roles of HSP70 in physiological and also pathological contexts $(3-5,34-35)$, we aimed here at re-addressing the question of $H S P A 1 A$ expression in peripheral blood immune cells at the mRNA level by real-time PCR and also regarding protein expression by flow cytometry.

Contrary to previous studies (14-16), HSPA1A expression was found to be upregulated in PBMCs from MS patients compared to HDs. When investigating the discrepancies across studies, it should be taken into account that in previous studies HSPA1A expression was determined with the microarray technology, and single gene validation of HSPA1A findings with more sensitive techniques, such as real-time PCR, 
was lacking in all cases. Differences between studies were not related to the sample storage conditions, as in the present study HSPA1A expression was comparable between fresh cells and cryopreserved samples. Other factors that could have contributed to the discrepancies may be related to the clinical characteristics of MS patients: i) In the present study patients never received immunomodulatory or immunosuppressant treatment, whereas in previous studies patients with a washout period of 1-2 months between treatment and sample collection were considered eligible for gene expression profiling; ii) In the present study, PBMCs were collected from MS patients with active disease activity that fulfilled the criteria for immunomodulatory treatment. In this regard, differences in the activation status of PBMCs may also explain the differences observed across studies.

As a next step, we analysed whether the up-regulation in HSPA1A gene expression also correlated with the protein expression levels. Cytoplasmic HSP70-1A/B protein expression levels were similar between MS patients and HDs. However, because HSP70 translocates to the nucleus following stress (22), we also determined the total (cytoplasmic plus nuclear) HSP70-1A/B protein expression. In line with the HSPA1A gene expression data, total HSP70-1A/B protein expression levels were significantly increased in T lymphocytes and monocytes from MS patients compared with HDs.

Although MS etiopathogenesis is not yet fully understood, multiple lines of evidence support that CD4+ T cells, but also other cell types including CD8+ T lymphocytes and monocytes play important roles in the pathogenesis of MS (36). In this regard, increased cellular levels of total HSP70-1A/B protein in PBMCs may be the result of stress associated with the immune cell activation that occurs in MS (reviewed in (3637)). Cwiklinska and co-workers (2010) did not find differences in basal HSP70 protein expression in PBMCs from MS patients and HDs using a commercial ELISA kit. However, these authors reported an aberrant induction of HSP70 protein expression in PBMCs of MS patients following an inflammatory or heat stress (20). Using a similar protocol, we assessed protein induction of total HSP70-1A/B in $T$ lymphocytes and 
monocytes following HS and LPS stresses. The HS stimulus induced a significant increase in HSP70-1A/B protein expression in CD4+ T lymphocytes from HDs, that reached similar levels to those of non-stimulated CD4+ T cells from MS patients. However, in MS patients the induction of HSP70-1A/B expression showed by CD4+ T lymphocytes following HS stimulus was only moderate compared with that of HDs. These data seem to indicate that HSP70-1A/B is chronically induced in CD4+ T cells from MS patients and, as a result, HS stimulus failed to trigger a significant HSP70$1 A / B$ induction. Interestingly, we also found that the levels of total HSP70-1A/B protein expression by heat-shocked monocytes correlated with the number of T2 lesions at baseline. This could be accounted by the fact that monocytes/macrophages are part of the inflammatory cuffs found in the CNS of MS patients and the T2-weighted MRI is commonly applied to quantify the accumulated MS lesion load. Furthermore, it has described that human HSPs might promote autoimmunity by peptidomimetic mechanism due to their similarity with bacteria HSP paralogs (38).

We also explore differences in HSP70-1A/B expression in monocytes stimulated with LPS. This stimulation primarily activates monocytes and consequently triggers HSP70$1 A / B$ expression in HDs and MS patients $(20,39-40)$. We found that monocytes from MS patients also exhibited reduced response to the inflammatory stimulus, since HSP70-1A/B up-regulation following LPS stimulation was restricted to the control population. The reasons behind this differential response to LPS stimulation in terms of HSP70-1A/B induction are unknown. However, it is tempting to speculate that the higher baseline HSP70-1A/B expression levels in monocytes from MS patients rendered the cells unresponsive to subsequent HSP70-1A/B-inducing stimuli. Other factors such a lower expression of TLR4, the LPS receptor, in monocytes from MS patients could also have contributed to these differential findings (41).

In summary, we found a baseline increased expression of HSPA1A gene in PBMCs from MS patients compared with HDs. Gene expression findings were associated with an increased protein expression of HSP70-1A/B in T lymphocytes (CD4+ and CD8+) 
and monocytes from MS patients under basal conditions that may reflect the immunological activation that occurs in MS patients. We also provided evidence that after a stress stimulus CD4+ $\mathrm{T}$ lymphocytes as well as monocytes from MS patients showed reduced induction of HSP70-1A/B protein expression. This points out that immune response is altered in MS and it may indicate a state of chronic stress in MS patients. These data suggest that strategies for reducing or inhibiting HSP70-1A/B expression may be beneficial for MS patients, and, in support of this possibility, it has been reported that HSP70-deficient mice are resistant to EAE (42). However, the side effects associated with the essential cytoprotective functions of HSP70-1A/B should be taken into account. 


\section{Acknowledgements}

We are grateful to the patients and healthy donors who agreed to participate in this study and to our nurses, Mrs. R. Horno and M.J. Vicente, for their efficient clinical/basic coordination in the provision of patient material. We thank the "Red Española de Esclerosis Múltiple (REEM)" (RD12/0032) - sponsored by the Fondo de Investigación Sanitaria (FIS), Ministry of Economy and Competition Spain - and the "Ajuts per donar Suport als Grups de Recerca de Catalunya (2009 SGR 0793)", sponsored by the "Agència de Gestió d’Ajuts Universitaris i de Recerca" (AGAUR), Generalitat de Catalunya, Spain. CE is partially supported by the "Miguel Servet" program (CP07/00146) from the FIS, Ministry of Economy and Competition, Spain.

\section{Declaration of Interest}

The authors declare that they have no conflict of interest. 
Figure Legends

Figure 1. HSP70 gene expression is increased in MS patients. (A) HSP70 (HSPA1A) relative gene expression was determined in total RNA from fresh PBMCs using real-time PCR, and PGK1 gene expression was used as an endogenous control. The results were normalised to the gene expression of HSP70 in HDs. MS patients showed 2.5-fold greater HSPA1A mRNA expression than HDs $(\mathrm{N}=30$ in each group, $p=0.038$ ). (B) In a subgroup of MS patients and HDs, HSPA1A relative gene expression was also determined following PBMC cryopreservation. No alterations in HSPA1A expression were detected in cryopreserved PBMCs from either MS or HD $(\mathrm{N}=9$ and $\mathrm{N}=10$, respectively). Error bars represent the standard error of the mean (SEM).

Figure 2. Total HSP70 protein expression is up-regulated in non-stimulated T lymphocytes and monocytes from MS patients. Cytoplasmic (A) and total (B) HSP70-1A/B protein expression was studied in CD4+ and CD8+ T lymphocytes and monocytes under baseline conditions. Although no differences were found in cytoplasmic HSP70-1A/B protein expression between groups $(\mathrm{N}=30 \mathrm{HD}$ and $\mathrm{N}=32$ MS patients), all cell types from MS patients showed increased total (cytoplasmic plus nuclear) HSP70-1A/B protein levels compared to HDs ( $\mathrm{N}=14$ in each group). Error bars represent SEM.

Figure 3. Induction of HSP70-1A/B protein expression following HS or LPS stimulus. Comparison of total HSP70-1A/B protein expression at baseline conditions and following HS in CD4+ and CD8+ T lymphocytes and in monocytes (A) or following LPS stimulus in monocytes (B) from MS patients and HDs ( $N=14$ in each group). Error bars represent SEM. 


\section{References}

1. Li, G. C., and Z. Werb. 1982. Correlation between synthesis of heat shock proteins and development of thermotolerance in Chinese hamster fibroblasts. Proceedings of the National Academy of Sciences of the United States of America 79: 3218-3222.

2. Riabowol, K. T., L. A. Mizzen, and W. J. Welch. 1988. Heat shock is lethal to fibroblasts microinjected with antibodies against hsp70. Science 242: 433-436.

3. Lindquist, S., and E. A. Craig. 1988. The heat-shock proteins. Annu Rev Genet 22: 631-677.

4. Welch, W. J. 1993. Heat shock proteins functioning as molecular chaperones: their roles in normal and stressed cells. Philos Trans $R$ Soc Lond B Biol Sci 339: 327-333.

5. Jaattela, M. 1999. Heat shock proteins as cellular lifeguards. Ann Med 31: 261271.

6. Hunt, C., and R. I. Morimoto. 1985. Conserved features of eukaryotic hsp70 genes revealed by comparison with the nucleotide sequence of human hsp70. Proceedings of the National Academy of Sciences of the United States of America 82: 6455-6459.

7. Tavaria, M., T. Gabriele, I. Kola, and R. L. Anderson. 1996. A hitchhiker's guide to the human Hsp70 family. Cell Stress Chaperones 1: 23-28.

8. Daugaard, M., M. Rohde, and M. Jaattela. 2007. The heat shock protein 70 family: Highly homologous proteins with overlapping and distinct functions. FEBS Lett 581: 3702-3710.

9. Li, Y., J. Subjeck, G. Yang, E. Repasky, and X. Y. Wang. 2006. Generation of anti-tumor immunity using mammalian heat shock protein 70 DNA vaccines for cancer immunotherapy. Vaccine 24: 5360-5370.

10. Gong, J., Y. Zhang, J. Durfee, D. Weng, C. Liu, S. Koido, B. Song, V. Apostolopoulos, and S. K. Calderwood. 2010. A heat shock protein 70-based vaccine with enhanced immunogenicity for clinical use. J Immunol 184: 488496.

11. Aquino, D. A., E. Capello, J. Weisstein, V. Sanders, C. Lopez, W. W. Tourtellotte, C. F. Brosnan, C. S. Raine, and W. T. Norton. 1997. Multiple sclerosis: altered expression of $70-$ and $27-\mathrm{kDa}$ heat shock proteins in lesions and myelin. Journal of neuropathology and experimental neurology 56: 664672.

12. Chabas, D., S. E. Baranzini, D. Mitchell, C. C. Bernard, S. R. Rittling, D. T. Denhardt, R. A. Sobel, C. Lock, M. Karpuj, R. Pedotti, R. Heller, J. R. Oksenberg, and L. Steinman. 2001. The influence of the proinflammatory cytokine, osteopontin, on autoimmune demyelinating disease. Science 294: 1731-1735.

13. Cwiklinska, H., M. P. Mycko, O. Luvsannorov, B. Walkowiak, C. F. Brosnan, C. S. Raine, and K. W. Selmaj. 2003. Heat shock protein 70 associations with myelin basic protein and proteolipid protein in multiple sclerosis brains. International immunology 15: 241-249.

14. Mycko, M. P., H. Cwiklinska, J. Szymanski, B. Szymanska, G. Kudla, L. Kilianek, A. Odyniec, C. F. Brosnan, and K. W. Selmaj. 2004. Inducible heat shock protein 70 promotes myelin autoantigen presentation by the HLA class II. J Immunol 172: 202-213.

15. Lund, B. T., Y. Chakryan, N. Ashikian, L. Mnatsakanyan, C. J. Bevan, R. Aguilera, T. Gallaher, and M. W. Jakowec. 2006. Association of MBP peptides with Hsp70 in normal appearing human white matter. Journal of the neurological sciences 249: 122-134.

16. Bomprezzi, R., M. Ringner, S. Kim, M. L. Bittner, J. Khan, Y. Chen, A. Elkahloun, A. Yu, B. Bielekova, P. S. Meltzer, R. Martin, H. F. McFarland, and 
J. M. Trent. 2003. Gene expression profile in multiple sclerosis patients and healthy controls: identifying pathways relevant to disease. Human molecular genetics 12: 2191-2199.

17. Mandel, M., M. Gurevich, R. Pauzner, N. Kaminski, and A. Achiron. 2004. Autoimmunity gene expression portrait: specific signature that intersects or differentiates between multiple sclerosis and systemic lupus erythematosus. Clinical and experimental immunology 138: 164-170.

18. Satoh, J., M. Nakanishi, F. Koike, S. Miyake, T. Yamamoto, M. Kawai, S. Kikuchi, K. Nomura, K. Yokoyama, K. Ota, T. Kanda, T. Fukazawa, and T. Yamamura. 2005. Microarray analysis identifies an aberrant expression of apoptosis and DNA damage-regulatory genes in multiple sclerosis. Neurobiology of disease 18: 537-550.

19. Comabella, M., and R. Martin. 2007. Genomics in multiple sclerosis--current state and future directions. Journal of neuroimmunology 187: 1-8.

20. Cwiklinska, H., M. P. Mycko, B. Szymanska, M. Matysiak, and K. W. Selmaj. 2010. Aberrant stress-induced Hsp70 expression in immune cells in multiple sclerosis. Journal of neuroscience research 88: 3102-3110.

21. Livak, K. J., and T. D. Schmittgen. 2001. Analysis of relative gene expression data using real-time quantitative PCR and the 2(-Delta Delta $\mathrm{C}(\mathrm{T})$ ) Method. Methods 25: 402-408.

22. Welch, W. J., and J. R. Feramisco. 1984. Nuclear and nucleolar localization of the 72,000-dalton heat shock protein in heat-shocked mammalian cells. The Journal of biological chemistry 259: 4501-4513.

23. Flynn, G. C., T. G. Chappell, and J. E. Rothman. 1989. Peptide binding and release by proteins implicated as catalysts of protein assembly. Science 245: 385-390.

24. Beckmann, R. P., L. E. Mizzen, and W. J. Welch. 1990. Interaction of Hsp 70 with newly synthesized proteins: implications for protein folding and assembly. Science 248: 850-854.

25. Hartl, F. U., and M. Hayer-Hartl. 2002. Molecular chaperones in the cytosol: from nascent chain to folded protein. Science 295: 1852-1858.

26. Mosser, D. D., and R. I. Morimoto. 2004. Molecular chaperones and the stress of oncogenesis. Oncogene 23: 2907-2918.

27. Benn, S. C., and C. J. Woolf. 2004. Adult neuron survival strategies--slamming on the brakes. Nature reviews. Neuroscience 5: 686-700.

28. Beere, H. M. 2004. "The stress of dying": the role of heat shock proteins in the regulation of apoptosis. Journal of cell science 117: 2641-2651.

29. Lanneau, D., A. de Thonel, S. Maurel, C. Didelot, and C. Garrido. 2007. Apoptosis versus cell differentiation: role of heat shock proteins HSP90, HSP70 and HSP27. Prion 1: 53-60.

30. Dyment, D. A., G. C. Ebers, and A. D. Sadovnick. 2004. Genetics of multiple sclerosis. Lancet neurology 3: 104-110.

31. Aquino, D. A., A. A. Klipfel, C. F. Brosnan, and W. T. Norton. 1993. The 70-kDa heat shock cognate protein (HSC70) is a major constituent of the central nervous system and is up-regulated only at the mRNA level in acute experimental autoimmune encephalomyelitis. Journal of neurochemistry 61: 1340-1348.

32. Bajramovic, J. J., M. Bsibsi, S. B. Geutskens, R. Hassankhan, K. C. Verhulst, G. J. Stege, C. J. de Groot, and J. M. van Noort. 2000. Differential expression of stress proteins in human adult astrocytes in response to cytokines. Journal of neuroimmunology 106: 14-22.

33. Mansilla, M. J., X. Montalban, and C. Espejo. 2012. Heat Shock Protein 70: Roles in Multiple Sclerosis. Mol Med. 
34. Tytell, M., and P. L. Hooper. 2001. Heat shock proteins: new keys to the development of cytoprotective therapies. Expert opinion on therapeutic targets 5: 267-287.

35. Turturici, G., G. Sconzo, and F. Geraci. 2011. Hsp70 and its molecular role in nervous system diseases. Biochemistry research international 2011: 618127.

36. Sospedra, M., and R. Martin. 2005. Immunology of multiple sclerosis. Annual review of immunology 23: 683-747.

37. Noseworthy, J. H., C. Lucchinetti, M. Rodriguez, and B. G. Weinshenker. 2000. Multiple sclerosis. The New England journal of medicine 343: 938-952.

38. Van Eden, W., G. Wick, S. Albani, and I. Cohen. 2007. Stress, heat shock proteins, and autoimmunity: how immune responses to heat shock proteins are to be used for the control of chronic inflammatory diseases. Ann N Y Acad Sci 1113: 217-237.

39. Edelman, D. A., Y. Jiang, J. G. Tyburski, R. F. Wilson, and C. P. Steffes. 2007. Lipopolysaccharide up-regulates heat shock protein expression in rat lung pericytes. The Journal of surgical research 140: 171-176.

40. Bernardini, C., A. Zannoni, M. L. Bacci, and M. Forni. 2010. Protective effect of carbon monoxide pre-conditioning on LPS-induced endothelial cell stress. Cell Stress Chaperones 15: 219-224.

41. Bustamante, M. F., N. Fissolo, J. Rio, C. Espejo, C. Costa, M. J. Mansilla, I. Lizasoain, M. A. Moro, M. Carmen Edo, X. Montalban, and M. Comabella. 2011. Implication of the Toll-like receptor 4 pathway in the response to interferon-beta in multiple sclerosis. Annals of neurology 70: 634-645.

42. Mycko, M. P., H. Cwiklinska, A. Walczak, C. Libert, C. S. Raine, and K. W. Selmaj. 2008. A heat shock protein gene (Hsp70.1) is critically involved in the generation of the immune response to myelin antigen. European journal of immunology 38: 1999-2013. 